\title{
El ajuste presupuestario en las Entidades Locales
}

\author{
José Francisco Bellod Redondo \\ Departamento de Economía \\ Universidad Politécnica de Cartagena
}

Sumario: I. INTRODUCCIÓN. II. EL RÉGIMEN DEL ENDEUDAMIENTO LOCAL. III. RAZÓN DE SER DE LA REFORMA. IV. RESULTADOS DEL AJUSTE PRESUPUESTARIO A NIVEL LOCAL. V. CONCLUSIONES.

\section{INTRODUCCIÓN}

Con ocasión del proceso de consolidación presupuestaria llevado a cabo en todos los niveles de la Administración española a partir de Tratado de Maastricht (y posteriormente con el Pacto de Estabilidad y Crecimiento), tuvo lugar la aprobación de un procedimiento de ajuste presupuestario en el ámbito de las Haciendas Locales.

Dicho proceso se implementó mediante el artículo 59 y la Disposición Transitoria Séptima de la Ley 50/1998, de 30 de diciembre, de Medidas Fiscales, Administrativas y de Orden Social. Dicha norma modificó el régimen de endeudamiento Local vigente hasta ese momento, condicionando las posibilidades, no sólo de endeudamiento sino también el gasto público de las Entidades sujetas a dicha legislación.

Posteriormente, la aprobación de la Ley 8/2001, de 12 de diciembre, General de Estabilidad Presupuestaria, que de modo expreso impone un límite al déficit público de las Entidades Locales, vendría a constatar las limitaciones de la reforma de 1998 en aras a contribuir al cumplimiento de los objetivos fijados en el Pacto de Estabilidad y Crecimiento.

En el presente trabajo analizamos con los datos disponibles, el impacto de la Ley 50/1998 sobre la evolución presupuestaria de las Entidades Locales. La ausencia de datos impide analizar por ahora el verdadero impacto de la Ley 8/2001.

\section{EL RÉGIMEN DEL ENDEUDAMIENTO LOCAL}

El régimen jurídico del endeudamiento de las Entidades Locales viene establecido en los artículos 51 y siguientes del Real Decreto Legislativo 
2/2004, de 5 de marzo, por el que se aprueba el Texto Refundido de la Ley Reguladora de las Haciendas Locales. Siguiendo el esquema original de la Ley 39/1988, de 29 de diciembre, reguladora de las Haciendas Locales (en adelante LRHL), el actual Texto Refundido establece un régimen dual para el endeudamiento distinguiendo entre Operaciones de Tesorería y Endeudamiento a Largo Plazo.

Las Operaciones de Tesorería están constituidas por el endeudamiento a corto plazo (igual o inferior a una año) y se justifican por los desfases que a lo largo del ejercicio presupuestario se producen entre los ingresos y los pagos presupuestados. Tienen naturaleza extrapresupuestaria y, en consecuencia no se registran en el capítulo 9 del presupuesto. Además, contrariamente al endeudamiento a largo plazo, los ingresos obtenidos de estas operaciones escapan al principio de afectación a la financiación de inversiones.

Las operaciones de Endeudamiento a largo plazo son aquellas que se conciertan por plazo superior al año y que tienen por finalidad financiar inversiones o sustituir total o parcialmente operaciones de crédito preexistentes.

En la redacción original de la LRHL se establecía un régimen específico para la autorización de cada uno de los tipos de operaciones citados. Así, las Entidades Locales podían concertar Operaciones Transitorias de Tesorería por plazo superior a un año siempre que su importe no superase el 30\% de los ingresos corrientes liquidados en el ejercicio inmediatamente anterior. En cuanto a las Operaciones de Crédito a Largo Plazo debían cumplir en primer lugar el «principio de afectación», esto es, tener por finalidad la financiación de inversiones o la reprogramación de créditos preexistentes ${ }^{1}$.

La reforma introducida mediante la Ley 50/1998, de 30 de diciembre, de Medidas Fiscales, Administrativas y de Orden Social viene a limitar cuantitativamente la capacidad de endeudamiento en el largo plazo de las Entidades Locales. A tal fin establece, en primer lugar un régimen de autorización más restrictivo que el preexistente. Así, requerirán autorización previa del Ministerio de Economía y Hacienda aquellas Operaciones de Crédito de Largo Plazo cuando el «Ahorro Neto» resultante de tener en cuenta la operación de crédito a concertar resulte negativo. En segundo lugar, resultando negativo el «Ahorro Neto» de la Entidad Local, ésta tiene la obligación de presentar un «Plan de Saneamiento Financiero» de plazo no superior a tres años para ajustar a cero dicha magnitud. En tercer lugar, también

\footnotetext{
1 Lo que suele conocerse como operaciones de «conversión de deuda».
} 
será preceptiva la autorización previa en aquellas operaciones de crédito en las que el capital vivo resultante (corto plazo + largo plazo) exceda del $110 \%$ de los ingresos corrientes liquidados en el ejercicio anterior ${ }^{2}$. A las Entidades Locales con una población superior a los 200.000 habitantes se les permitía la posibilidad de sustituir el «Plan de Saneamiento Financiero» por un «Escenario de Consolidación presupuestaria». En síntesis la diferencia entre el «Plan de Saneamiento Financiero» y el «Escenario de Consolidación presupuestaria» consiste en que el primero debía ser aprobado por el Ministerio de Economía y Hacienda junto a la solicitud de la nueva operación de crédito, mientras que el segundo requería exclusivamente la aprobación del Pleno de la Entidad local.

La Disposición Transitoria Séptima de la Ley 50/1998 venía a flexibilizar el severo régimen de endeudamiento introducido mediante el artículo 59 de dicha Ley. Así, se establecía la innecesariedad de solicitar autorización para concertar operaciones de crédito a largo plazo en los años 1999 y 2000 siempre que el ahorro neto aún siendo de signo negativo no supere los siguientes porcentajes en relación con los ingresos corrientes liquidados: -1'50\% en el año 1999, y el - $0^{\prime} 75 \%$ en el ejercicio 2000.

El empleo de este tipo de normas que trata de controlar la evolución del déficit público mediante la imposición de límites cuantitativos (en este caso la ratio del $110 \%$ Deuda / Ingresos Corrientes), pertenece a lo que se ha dado en llamar instituciones presupuestarias de «naturaleza continental», por ser propias de los países de la Europa continental, frente a la tradición anglosajona, que trata de controlar el déficit público mediante regulaciones en «el modo» de elaborar o ejecutar el presupuesto. Aunque ambos tipos de normas tienen como trasfondo común el de tratar de garantizar la sostenibilidad de las finanzas públicas, aún existe un amplio debate acerca de la idoneidad de ambos tipos y en el caso español es común encontrar elementos procedentes de ambas tradiciones si bien las normas de naturaleza continental están mucho más arraigadas ${ }^{3}$

\section{RAZÓN DE SER DE LA REFORMA}

¿Cuál era la pretensión de la reforma del endeudamiento local introducida mediante la Ley 50/1998?

\footnotetext{
${ }^{2}$ Artículo 59.1 de la Ley 50/1998, de 30 de diciembre, de Medidas Fiscales, Administrativas y de Orden Social.

${ }^{3}$ Entre los análisis referidos a la sostenibilidad presupuestaria en el ámbito de las Entidades Locales cabe destacar el trabajo de I. Ezquiaga (2000), «Condiciones de la Sostenibilidad Financiera de las Corporaciones Locales 1999 - 1999», en Papeles de Economía Española, no 83.
} 
Básicamente se pretendía incorporar a las Entidades Locales al proceso de ajuste presupuestario que habría de conducir al cumplimiento de las condiciones necesarias para acceder a la última fase de la Unión Económica y Monetaria. El Pacto de Estabilidad y Crecimiento, acordado en la Cumbre de Ámsterdam en 1997, «limita la utilización del déficit público como instrumento de política económica de la Unión Económica y Monetaria. En virtud del referido Pacto, los Estados miembros de la Unión se comprometen a perseguir el objetivo a medio plazo de situaciones presupuestarias próximas al equilibrio o en superávit, de forma que, cuando los estabilizadores automáticos actúen, el déficit se mantenga siempre por debajo del $3 \%$. Los Estados también se comprometen a presentar anualmente Programas de Estabilidad o Convergencia que recojan las medidas necesarias para alcanzar ese objetivo» ${ }^{4}$. En concreto, el Pacto de Estabilidad y Crecimiento obliga a los países miembro de la Unión Europea a garantizar que sus déficit públicos respectivos se sitúen por debajo del $3 \%$, con la perspectiva de lograr el equilibrio presupuestario en el medio plazo. Es, en consecuencia, un mandato más restrictivo que el que se establece en el Tratado de Maastricht.

Aceptado el resultado de la Cumbre de Ámsterdam, sólo queda repartir el «sacrificio presupuestario» entre los diversos niveles de cada administración. En el caso español, se trata de limitar el déficit público y el endeudamiento de las Administraciones (Central, Autonómica y Local). En otras palabras, la Ley 50/1998 fue el instrumento jurídico por medio del cual se instrumentó la contribución de las Entidades Locales a la consecución de los objetivos fijados en la Cumbre de Ámsterdam.

¿Cuál es la conexión formal entre la reforma introducida por la Ley 50/1998 y los objetivos de contención del déficit público establecidos en el Pacto de Estabilidad y Crecimiento?

Como indica el artículo 165.4 del texto Refundido de la LRHL ${ }^{5}$, «cada uno de los presupuestos que se integran en el Presupuesto General [de las Entidades Locales] deben aprobarse sin déficit inicial». Se refiere la norma al déficit como diferencia entre la totalidad de ingresos y la totalidad de gastos inicialmente presupuestados, incluidos los ingresos procedentes de la emisión de pasivos financieros, y no al déficit público como necesidad de financiación de la entidad. Es decir, el déficit público (necesidad de financiación) debe quedar inicialmente cubierto con la emisión

\footnotetext{
4 Preámbulo de la Ley 18/2001, de 12 de diciembre, General de Estabilidad Presupuestaria.

5 Artículo 146.4 de la LRHL.
} 
de pasivos financieros (capítulo 9 del presupuesto) o mediante la liquidación de activos financieros (capítulo 8 del presupuesto). Como lo habitual es que las Entidades Locales dispongan de una cartera muy reducida (o nula) de activos financieros, podemos simplificar la cuestión señalando que la Ley obliga a financiar el déficit público mediante la variación neta de pasivos financieros, es decir, mediante la diferencia entre lo que se va a ingresar por la concertación de operaciones de crédito a largo plazo (capítulo 9 del presupuesto de ingresos) y lo que se va a pagar en concepto de amortización de esos mismos créditos (capítulo 9 del presupuesto de gastos), esto es:

$$
d p_{t}=-v n p f_{t}
$$

es decir, el déficit público $d p_{t}$ debe ser de igual magnitud, pero de signo contrario, que la variación neta de pasivos financieros $v n p f_{t}$ siendo la diferencia entre la suma de los ingresos de los capítulos 1 a 7 del presupuesto de ingresos y los análogos del presupuesto de gastos. Si la variable $d p_{t}$ presenta un importe negativo estaremos en presencia de déficit público y será necesario aumentar el endeudamiento a largo plazo, si su importe es positivo tendremos superávit público y ello permitirá amortizar deuda pública. Denominemos $B_{t}$ al importe vivo de la deuda (corto plazo + largo plazo) de una corporación local a 31 de diciembre del año «t». Sea $I{ }_{t}$ los ingresos procedente del capítulo 9 (Pasivos Financieros) en el ejercicio «t», es decir, la contratación de nuevos créditos a largo plazo; sea $G 9$ t la amortización de créditos de largo plazo durante el ejercicio «t» (capítulo 9 del presupuesto de gastos), y sea $\Delta \delta$ la variación del saldo de Operaciones de Tesorería, entonces debe cumplirse que:

$$
B_{t}=B_{t-1}+I 9_{t}-G 9_{t}+\Delta \delta
$$

es decir, la deuda al final de un ejercicio dado «t», es igual a la que había al final del ejercicio precedente «t-1», más la nueva deuda contratada $I{ }_{t}$ , menos la deuda amortizada , más el incremento (positivo o negativo) operado en la deuda a corto plazo por operaciones de tesorería . Al limitar el volumen de la deuda al $110 \%$ del importe de los Ingresos Corrientes liquidados en el ejercicio anterior $\left(I C_{1-t}\right)$ estamos imponiendo la condición:

$$
B_{t} \leq 1^{\prime} 10 \cdot I C_{t-1}
$$

sustituyendo la ecuación [3] en la ecuación [2] y reordenando obtenemos:

$$
I 9_{t}-G 9_{t} \leq 1^{\prime} 10 \cdot I C_{t-1}-B_{t-1}-\Delta \delta
$$


El término a la izquierda de la ecuación [4] es la denominada variación neta de pasivos financieros $\left(v n p f_{t}\right)$, lo cual nos permite sustituir la ecuación [1] en [4] quedando finalmente:

$$
d p_{t} \geq 1^{\prime} 10 \cdot I C_{t-1}-B_{t-1}-\Delta \delta
$$

La ecuación [5] demuestra la vinculación entre la limitación de la ratio Deuda / Ingresos Corrientes y el control del déficit público. Al establecer un ratio máximo de endeudamiento del 110\%, la Ley 50/1998 está en realidad limitando cuantitativamente el importe del déficit público. Además, el importe del déficit público de las Entidades Locales está limitado por el importe de los derechos reconocidos por Ingresos Corrientes en el ejercicio precedente; por el importe de la deuda (corto plazo + largo plazo) existente al inicio del ejercicio y por la variación experimentada por las Operaciones de Tesorería durante el ejercicio. Concretamente, el déficit público en el que puede incurrir una Entidad Local será mayor cuanto mayor haya sido el importe de los Ingresos Corrientes liquidados en el ejercicio precedente, y menor cuanto mayores hayan sido el importe de la deuda con la que se inició el ejercicio o la variación en el saldo de las operaciones de tesorería.

\section{RESULTADOS DEL AJUSTE PRESUPUESTARIO A NIVEL LOCAL}

Para tratar de avaluar el impacto que las reformas legales citadas han tenido en el ámbito local, podemos recurrir a diversos indicadores. En el Cuadro 1 hemos incluido la Capacidad ó Necesidad de Financiación de las diversas Administraciones Públicas. Estos datos han sido confeccionados por el Banco de España de acuerdo con el sistema contable SEC95. La Capacidad / Necesidad de Financiación es la medida que mejor recoge el importe del déficit público tal y como habitualmente lo definimos, esto es, la diferencia entre los ingresos procedentes de los siete primeros capítulos del presupuesto de ingresos y los gastos de los siete primeros capítulos del presupuesto de gastos. Cuando la magnitud es negativa esa entidad presenta Necesidad de Financiación y requerirá liquidar Activos Financieros (capítulo 8) ó concertar nuevas operaciones de crédito (capítulo 9).

Como puede comprobarse en el Cuadro 1, en los años previos a la aprobación de la reforma introducida por la Ley 50/1998, las Corporaciones Locales presentaban, a nivel agregado, un resultado muy satisfactorio en términos de capacidad de financiación. Así, por ejemplo, en $1996 \mathrm{su}$ 


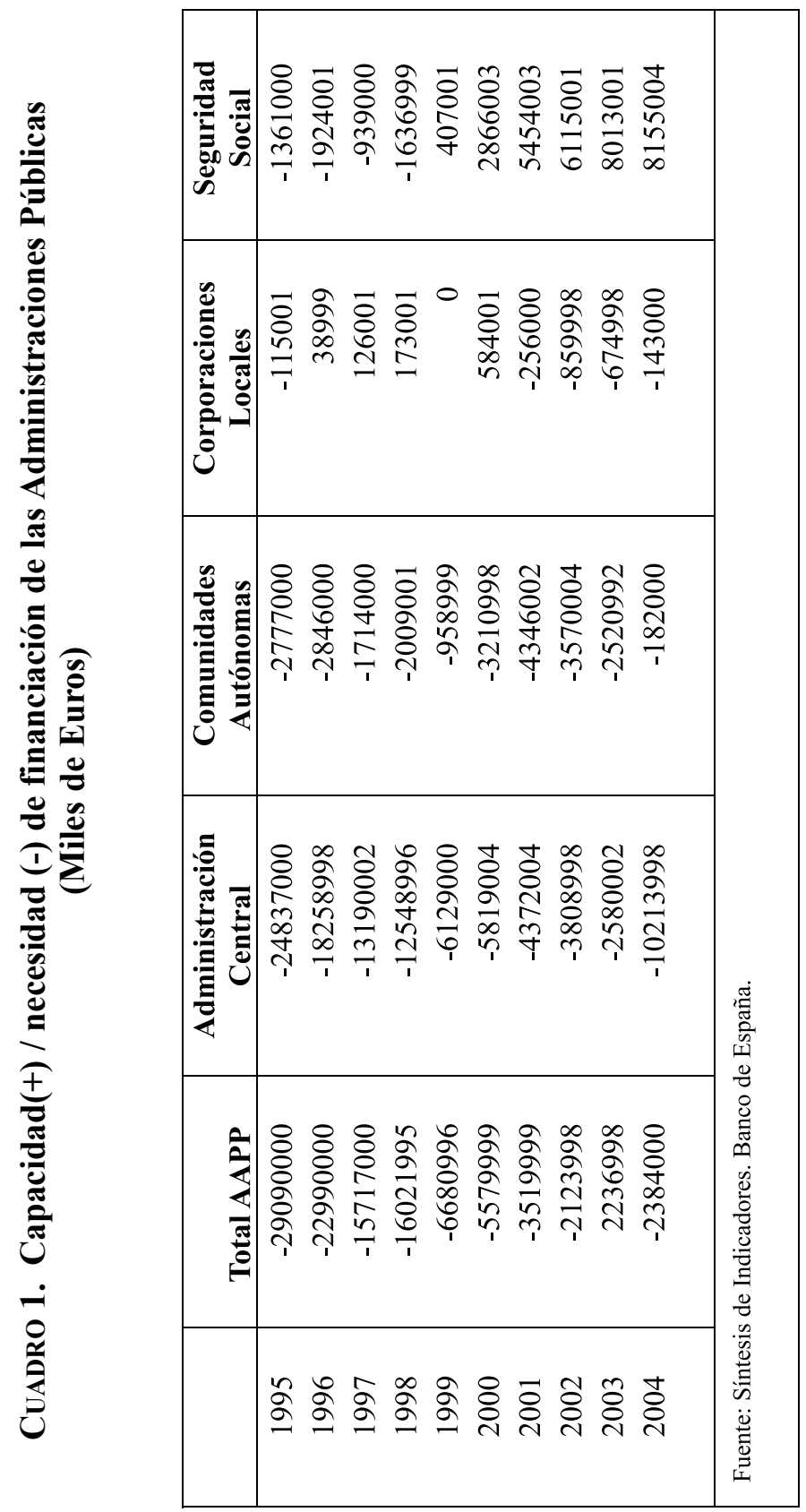


Capacidad de Financiación ascendió a 38.999 miles de euros, a 126.001 miles de euros en 1997 y a 173.001 miles de euros en 1998. Aunque en 1999, primer año de vigencia de la reforma no se registró ni capacidad ni necesidad de Financiación, en el año 2000 se registró la cifra record de 584.001 miles de euros. Estos resultados son especialmente meritorios si los comparamos con los registros que ofrecen el resto de Administraciones Públicas. Sólo la Seguridad Social, debido a la etapa de expansión de la renta y el empleo que caracterizó el período 2000 - 2004, presenta sistemáticamente capacidad de Financiación y además creciente, llegando a la cifra record de 8.155.004 miles de euros en el ejercicio 2004. Comunidades Autónomas y Administración Central resultan deficitarias en todos el periodo estudiado, si bien puede apreciarse la tendencia a la baja seguida en ambas.

Otro dato de interés es la evolución del endeudamiento acumulado de las distintas administraciones públicas. En el Cuadro 2 hemos registrado el importe de la deuda pendiente a 31 de diciembre, para cada una de las Administraciones en el período 1995 - 2004. Estos datos son confeccionados por el Banco de España de acuerdo con el Protocolo de Déficit Excesivo (incluido en el Pacto de Estabilidad y Crecimiento). Puede comprobarse que el endeudamiento de las Corporaciones Locales resulta francamente residual en el conjunto de del endeudamiento público, oscilando entre el 6'4\% del año 1995 y el 5'3\% registrado en los años 2000 y 2001.

Ciertamente con anterioridad a la aprobación de la Ley 50/1998 el endeudamiento de las Corporaciones Locales estaba creciendo en términos absolutos, de hecho pasó de 17.941.074 miles de euros en 1995 a 19.093.192 miles de euros en 1998. Sin embargo su crecimiento era muy moderado en comparación con el de la Administración Central o las Comunidades Autónomas. Ello ha permitido que, incluso antes de aprobarse la Ley 50/1998 la participación del endeudamiento local en el del total de las Administraciones Públicas se redujese paulatinamente, pasando del 6'4\% de 1995 al 5'6\% de 1998. Ello nos lleva a dudar de la necesidad de la Ley 50/1998 como instrumento incentivador del ajuste fiscal. En otras palabras: contrariamente a lo que sucedía con el resto de Administraciones, las Corporaciones Locales estaban cumpliendo sus deberes aún con anterioridad a la llamada de atención que supuso reforma aprobada en 1998. Y si el comportamiento anterior a 1998 nos hace dudar de su necesidad, el comportamiento posterior nos conduce a dudar de su eficacia. A partir del año 2002 se produce un repunte del endeudamiento local a un ritmo superior que el del conjunto de las Administraciones Públicas, de modo que ya en el año 2004 el peso del endeudamiento local se sitúa en el 6'2\%, es decir, muy próximo al registrado en el año 1995. 


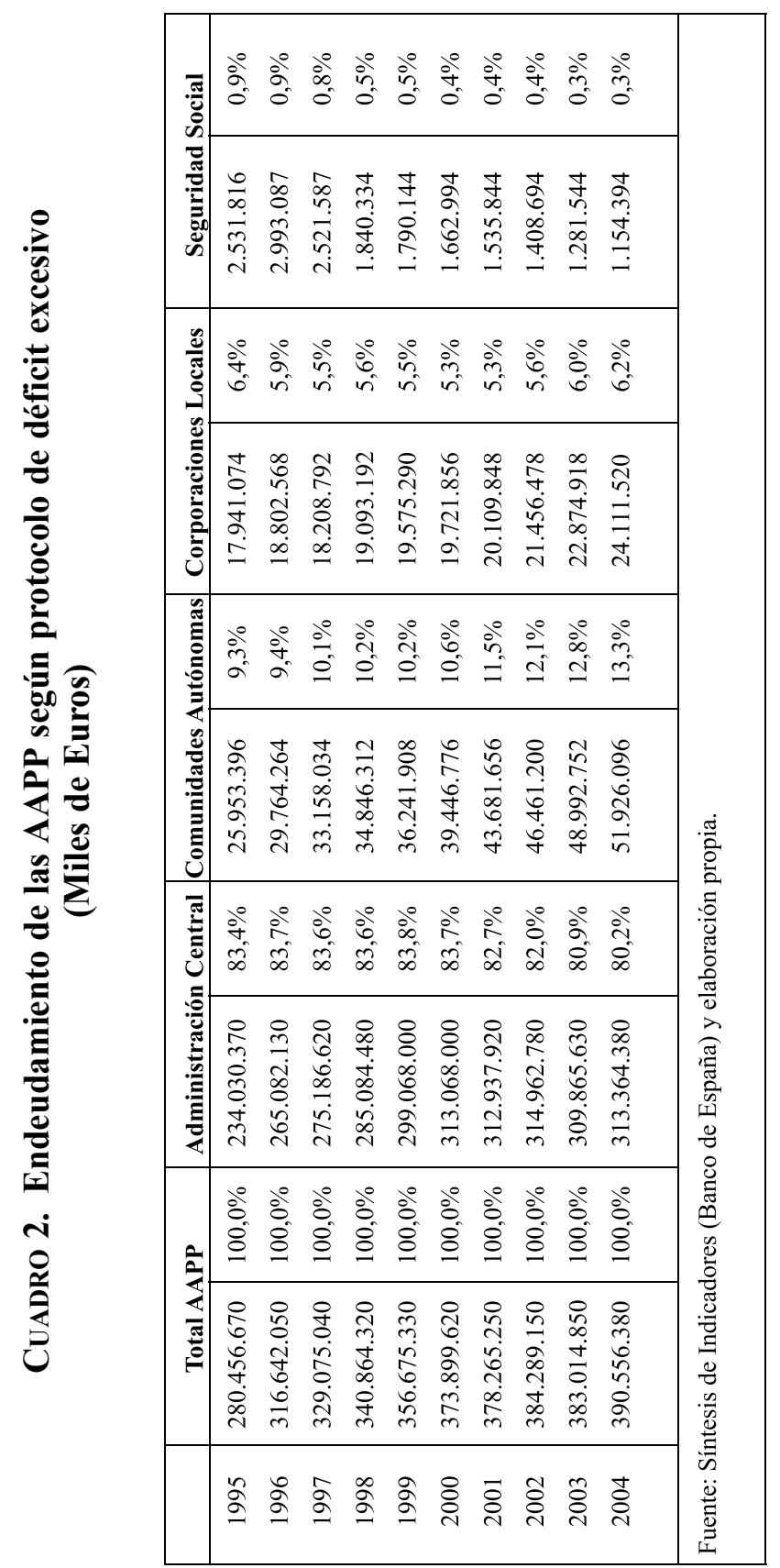


En otras palabras, la Ley 50/1998 no ha sido eficaz en el tiempo a efectos de limitar la evolución del endeudamiento local.

¿Cuál ha sido entonces la contribución de la Ley 50/1998 a la evolución del endeudamiento de las Corporaciones Locales? En el Cuadro 3 hemos registrado, para el periodo 1995 - 2001, el importe de la deuda acumulada de las Corporaciones Locales y el importe de los Ingresos Corrientes (derechos reconocidos). Los datos proceden de la base de datos BADESPE, del Instituto de Estudios Fiscales, dependiente del Ministerio de Hacienda, y de la Síntesis de Indicadores confeccionada por el Banco de España. En la última columna aparece la proporción entre el endeudamiento y los Ingresos corrientes. Puede comprobarse nuevamente que ya desde 1995 se venía operando una clara tendencia a la reducción de la ratio Deuda / Ingresos Corrientes. A nivel agregado puede comprobarse incluso que los ratios registrados en el periodo son muy inferiores al $110 \%$ que la Ley 50/1998 establece como límite al endeudamiento. Obviamente estos datos resultan de la agregación del conjunto de las Corporaciones Locales y es inmediato suponer que en todo ese periodo habrán existido (e incluso existirán hoy día) entidades que presenten ratios de endeudamiento superiores e inferiores a los registrados en el Cuadro 3 que, al fin y al cabo, viene a ser la media del ratio de endeudamiento de las Corporaciones Locales.

Si embargo, el valor registrado en el Cuadro 3 sí resulta ilustrativo de la tendencia operada de modo que, a falta de la publicación de datos desagregados por parte de las instituciones competentes, puede afirmarse que, salvo excepciones, las Corporaciones Locales, en conjunto, han reducido sustancialmente su ratio de endeudamiento en el periodo 1995 - 2001.

Cosa distinta es que esa reducción haya tenido lugar gracias a las limitaciones introducidas en 1998. De hecho en el Cuadro 4 se nos ofrecen evidencias que nos informan de otras tendencias que ha resultado ser determinantes en la evolución del endeudamiento local. El Cuadro 4 es análogo al Cuadro 3, sólo que ahora hemos registrado en porcentaje el crecimiento interanual de la deuda vida y de los Ingresos Corrientes. Un dato llama la atención: el volumen de deuda ha crecido por término medio a un ritmo del 2'0\% anual, mientras que los Ingresos Corrientes lo han hecho al 7'4\%. Ello explica el por qué de la reducción del ratio de endeudamiento a pesar de observarse un volumen creciente de endeudamiento local: el dinamismo de la economía en estos años, que se ha traducido en un importante crecimiento de los Ingresos Corrientes ha permitido a las Corporaciones Locales alejarse «a la baja» de la limitación del 110\% impuesta por la reforma del endeudamiento local de 1998. 
CuAdro 3. Endeudamiento de las Corporaciones Locales (Miles de Euros)

\begin{tabular}{|c|c|c|c|}
\hline & Deuda & Ingresos Corrientes & $\%$ \\
\hline 1995 & 17.941 .074 & 24.493 .906 & $73,2 \%$ \\
1996 & 18.802 .568 & 26.542 .648 & $70,8 \%$ \\
1997 & 18.208 .792 & 28.663 .842 & $63,5 \%$ \\
1998 & 19.093 .192 & 30.864 .189 & $61,9 \%$ \\
1999 & 19.575 .290 & 32.932 .849 & $59,4 \%$ \\
2000 & 19.721 .856 & 35.459 .299 & $55,6 \%$ \\
2001 & 20.109 .848 & 37.544 .763 & $53,6 \%$ \\
\hline \multicolumn{4}{|l|}{} \\
\hline \multicolumn{4}{|l|}{ elabonte: BADESPE (IEF), Síntesis de Indicadores (Banco de España) y } \\
\hline
\end{tabular}

CuAdro 4. Endeudamiento de las Corporaciones Locales (Miles de Euros)

\begin{tabular}{|c|c|c|c|c|}
\hline & Deuda & $\Delta \%$ & $\begin{array}{c}\text { Ingresos } \\
\text { Corrientes }\end{array}$ & $\Delta \%$ \\
\hline 1995 & 17.941 .074 & & 24.493 .906 & \\
1996 & 18.802 .568 & $4,8 \%$ & 26.542 .648 & $8,4 \%$ \\
1997 & 18.208 .792 & $-3,2 \%$ & 28.663 .842 & $8,0 \%$ \\
1998 & 19.093 .192 & $4,9 \%$ & 30.864 .189 & $7,7 \%$ \\
1999 & 19.575 .290 & $2,5 \%$ & 32.932 .849 & $6,7 \%$ \\
2000 & 19.721 .856 & $0,7 \%$ & 35.459 .299 & $7,7 \%$ \\
2001 & 20.109 .848 & $2,0 \%$ & 37.544 .763 & $5,9 \%$ \\
\multicolumn{5}{|l}{} \\
Media & $2 \% 0 \%$ & $7{ }^{\prime} 4 \%$ \\
\hline \multicolumn{7}{|l}{ Fuente: BADESPE (IEF), Síntesis de Indicadores (Banco de España) y elaboración propia. } \\
\hline
\end{tabular}

\section{CONCLUSIONES}

El proceso de convergencia nominal con la Unión Europea ha dado lugar a un proceso de ajuste presupuestario en todos los ámbitos de la Administración Pública española, con el objetivo de cumplir los requisitos de moderación y equilibrio presupuestario fijados en el Pacto de Estabilidad y Crecimiento (Ámsterdam, 1997). 
En el caso de las Entidades Locales dos han sido las normas que directamente han promovido el ajuste presupuestario: la Ley 50/1998, de 30 de diciembre, de Medidas Fiscales, Administrativas y de Orden Social, y la Ley 18/2001, de 12 de diciembre, General de Estabilidad Presupuestaria. Siendo aún pronto para evaluar el impacto de la segunda de estas normas, sí que podemos apuntar algunas conclusiones respecto al impacto de la primera de ellas, que de modo indirecto limita el importe en del déficit público en el que pueden incurrir las Entidades Locales.

La modificación del régimen de endeudamiento a largo plazo introducida por la Ley 50/1998 no ha sido necesaria ni eficaz si consideramos las Entidades Locales en su conjunto. A esta conclusión llegamos tras comprobar que las citadas Entidades Locales se hallaban inmersas en un proceso de reducción de sus ratios de endeudamiento con anterioridad al ejercicio 1998. La ineficacia se comprueba al constatar que con posterioridad al año 2000 aumentan tanto la Necesidad de Financiación como el peso del Endeudamiento de estas Administraciones. La aprobación de la Ley 18/2001 es la constatación de la ineficacia de la Ley 50/1998 para impulsar, en el ámbito de las Entidades Locales, los objetivos plasmados en el pacto de Estabilidad y Crecimiento. 


\section{Reseñas de legislación y jurisprudencia}

